\title{
3D-Lab: a collaborative web-based platform for molecular modeling
}

\begin{abstract}
Aim: The use of 3D information has shown impact in numerous applications in drug design. However, it is often under-utilized and traditionally limited to specialists. We want to change that, and present an approach making 3D information and molecular modeling accessible and easy-to-use 'for the people'. Methodology/results: A userfriendly and collaborative web-based platform (3D-Lab) for 3D modeling, including a blazingly fast virtual screening capability, was developed. 3D-Lab provides an interface to automatic molecular modeling, like conformer generation, ligand alignments, molecular dockings and simple quantum chemistry protocols. 3D-Lab is designed to be modular, and to facilitate sharing of $3 \mathrm{D}$-information to promote interactions between drug designers. Recent enhancements to our open-source virtual reality tool Molecular Rift are described. Conclusion: The integrated drug-design platform allows drug designers to instantaneously access 3D information and readily apply advanced and automated 3D molecular modeling tasks, with the aim to improve decision-making in drug design projects.
\end{abstract}

First draft submitted: 12 April 2016; Accepted for publication: 7 July 2016;

Published online: 31 August 2016

Keywords: 3D visualization $\bullet$ drug design $\bullet$ high-performance computing $\bullet$ molecular modeling $\bullet$ open source $\bullet$ usability $\bullet$ virtual reality $\bullet$ virtual screening

All molecules ranging from structurally simple drugs like aspirin to complex systems like proteins are $3 \mathrm{D}$ objects. Knowledge about their 3D structure is important in most stages of drug discovery as it, for example, provides detailed information about ligand-protein interactions and conformational preferences, among other things.

Around 150 years ago, Le Bel and van't Hoff proved the concept of molecules as $3 \mathrm{D}$ objects [1] and thus changed the way of how scientists think about small and large molecules. Since then, experimental and theoretical methods have been developed and used to generate 3D information, which include $\mathrm{x}$-ray crystallography, NMR spectroscopy, homology modeling, molecular docking and conformational sampling. The course of using $3 \mathrm{D}$ information to shape design ideas is, however, still complex. First, raw data from experiments (e.g., x-ray or NMR) have to be processed. For example, preparing a protein for structure-based design studies [2] includes the following steps: splitting a flat (PDB) file into components; preparing amino acids by adding hydrogen and missing side-chains; assigning tautomeric forms and protonation states. In a similar fashion, ligand structures also need attention (e.g., assigning bondorders, protonation states and optimizing distorted bond lengths/angles). Beyond that, there is the challenge of adequately treating water in computational drug design [3]. This processing of $3 \mathrm{D}$ raw data has largely been automated. But parts still require manual intervention best suited for specialists. Second, 3D modeling is more complex than $1 \mathrm{D} / 2 \mathrm{D}$ methods. That is, when going from
Christoph Grebner', Magnus Norrby', Jonatan Enström', Ingemar Nilsson', Anders Hogner', Jonas Henriksson², Johan Westin², Farzad Faramarzi ${ }^{2}$, Philip Werner ${ }^{2}$ \& Jonas Boström*,1 ${ }^{1}$ Medicinal Chemistry Department, CVMD iMED, AstraZeneca, S-431 83 Mölndal, Sweden ${ }^{2}$ Research Discovery Informatics, AstraZeneca, Mölndal, S-431 83 Mölndal, Sweden *Author for correspondence: Tel.: +46317065251 Fax: +4631776 3710 jonas.bostrom@astrazeneca.com 
$2 \mathrm{D}$ to $3 \mathrm{D}$, molecules are no longer represented by formulas or flat structures but defined as 3D objects. Therefore, low-energy conformations need to be created. As most molecules relevant to drug design include several rotational bonds [4], enumerating and searching all relevant (low-energy) conformations quickly leads to the so-called combinatorial explosion. Correctly orienting the generated conformations in $3 \mathrm{D}$ space is another complicating factor.

Structure and ligand-based methods typically require sophisticated methods to rank-order datasets. For example, shape-based virtual screening and docking protocols need to be properly prepared and validated [5]. It goes without saying that these kinds of protocols have to be created by trained computational chemists to avoid common pitfalls [6]. However, once an established protocol (e.g., docking or shapematching against a putative bioactive conformation) has been established it can be advantageous to share it with others through, say, a web interface. It should be noted that we use the term protocol and work-flow interchangeably for the process of preparing input, performing calculations and making the results available, say, in a webpage. Making modeling work-flows automated and available through well-designed tools opens up for a more efficient usage and a larger audience; basically, any scientist (specialist and nonspecialist) can apply advanced computational drug design methods in that way. In this context it is important to stress that automated protocols are not intended to be used in isolation. Rather the contrary, the protocols should provide an interface between drug discovery scientists to facilitate collaborative work and allow faster and better decision-making. As a bonus, automation should free up time for the specialist to tackle other and possibly more creative tasks.

The scope of medicinal chemistry has changed over the years [7], and modeling is being democratized. For example, job descriptions for chemists and computational chemists are overlapping more and more, and chemists are frequently performing modeling work. As a consequence the nonspecialists (chemists) need access to user-friendly tools to help drive design. There are emerging commercial solutions (e.g., Biovia [8], EnginFrame [9], LiveDesign [10], Optibrium [11]) as well as proprietary tools (FOCUS) [12] aiming to close that gap. At AstraZeneca there have been previous efforts, although leaving room for improvements, mainly due to technological reasons. Thus it was decided to develop a simple and powerful environment, with a strong focus on usability to better exploit 3D information in drug design. None of the commercially available tools were judged to be adequate for our needs and limited software budget, resulting in in-house development.
In-house method development and the acquisition of commercial solutions both have their pros and cons. A commercial solution provides access to advanced libraries with different features and functions, supported by professionals (e.g., software developers and trained application scientists). However, licenses can be costly, and requests for customized implementations toward specific needs may linger, slowing down innovation. Furthermore, it is often forgotten that commercial solutions require maintenance, development and integration into corporate infrastructures. The latter represents a hurdle as infrastructures are usually complex (e.g., network, databases, data formats, file structures, and so on), and are never identical between different companies. Therefore, a commercial solution cannot currently be expected to simply be installed and immediately up and running, but requires a substantial amount of work to be seamlessly integrated. With the recent and exciting progress of digitalization and cloud technology the situation is, however, likely to change in the future.

An advantage of developing a tool internally is that it can be directly tailored toward the needs of its intended user base, and designed specifically to fit the infrastructure at hand. Short communication ways and fewer decision bodies facilitate fast development cycles and an improved tool designed for companies' specific requirements may be achieved. Of course, internal development may also make use of commercially available software development kits. Here, new web-based $3 \mathrm{D}$ visualizer software development kits can be seen as a representative example for building rich, dynamic and attractive web-based tools. In general, modeling in a browser has been difficult. But browser technology has recently taken off, and is now more performant. In contrast to stand-alone solutions, a web-based tool offers the advantage to be accessible from everywhere, and developing a web-based tool increases the portability due to standardization toward HTML/ CSS (HyperText Markup Language/Cascading Style Sheets).

The main focus was on developing a powerful, userfriendly and collaborative web-based tool that facilitates the use of $3 \mathrm{D}$ information and various modeling protocols from one central place. We call it 3D-Lab. In the current paper, we will concentrate on highlighting key features and concepts of 3D-Lab and present our implementation of the game-changing and blazingly fast, Google-like 3D virtual screening capability, based on the FastROCS technology [13]. Following our vision to bring 3D molecular modeling to nonspecialists ('the people'), we will conclude with recent developments on our virtual reality molecular viewer Molecular Rift [14]. 


\section{D-Lab integration \& implementation}

3D-Lab was developed as a web-based interface to make it easily available for every scientist within AstraZeneca from everywhere. Thus, one benefit of 3D-Lab is that it brings $3 \mathrm{D}$ molecular modeling to all people involved in drug hunting projects, and allows for (near) real-time modeling. The tool is intended to serve as an interface between, say, the expert computational chemists and the medicinal chemists facilitating interactions and speeding up decision-making. For example, calculations can be performed and discussed interactively during a drug design meeting, as compared with the traditional way of scheduling new meetings and by that doing postponing design decisions and progress.

We consider the key parameters for a successful integration and acceptance to be: high usability by an intuitive web interface; a fully modular implementation to allow integration of any type of modeling tool from any third-party software vendor; a collaborative design for instantaneous sharing and integration of data; and a state-of-the-art molecular visualization system. The primary focus of 3D-Lab is to allow for easy and intuitive real-time modeling, but it can also be used for longer batch jobs using high-performance computing (HPC). Thus, 3D-Lab is fully integrated in the HPC facilities of AstraZeneca allowing direct submission of calculations to large computer clusters, including scheduling for high throughput of a large number of jobs. By integrating the AstraZeneca data environment, direct access to stored molecule information (experimental or predicted) is also available. Furthermore, the chosen design of 3D-Lab will allow integration of cloud computing resources without interfering with the user experience. This makes 3D-Lab flexible and fit for upcoming changes in HPC environments.

3D-Lab is built using python code and is augmented by functions of commercial libraries from OpenEye [15], in particular the JavaScript 3D web viewer (see the 'JavaScript 3D web visualizer' section). With respect to development (e.g., the web-interface and web-services), a small and dedicated team of skilled on-site programmers (from the IT department) and experienced modelers (from research) were allowed to act autonomously to deliver 3D-Lab. In this manner, new relevant features could easily be added while rapid testing and instant feedback was ensured. This allowed achieving fast development cycles as well as quickly resolving technical issues, often without the end-users noticing a problem. The creative working environment and close cooperation between scientist and developers proved to be useful.

\section{D-Lab's three main modes}

The tool is divided into three main modes: admin mode; input mode; and output mode (see Figure 1). The idea is that as soon as the specialist has developed a validated protocol, it should be made available in 3D-Lab via the admin mode. Hence, new protocols can expediently be created there as well as existing protocols may be modified (and deleted). To the date of this publication, 3D-Lab provides established and tested models for several 3D protocols for molecular dockings (Glide [16], Gold [17] and Fred [18]), shape-based molecular alignment (ROCS [19]), custom models for quantum mechanics (QM) optimization and calculating hydrogen bond donor and acceptor strengths [20], using Gaussian [21] as well as various conformation generation protocols using OMEGA [22]. Each method comes with predefined settings, such as environment variables and required libraries loaded, and those are subsequently reused when a user is creating a new protocol. When making and publishing a new model, the user has the option to select where it will end up under 'Playground', 'DMPK', 'Safety' or under specific 'Projects'. Upon creation, the model is immediately made available to all users from the input mode. Figure 2 shows an overview of the admin page.

In the input mode, all available protocols are displayed in a pull-down menu, under the 'Playground', 'DMPK', 'Safety', 'Projects' and 'Conformational Models' subcategories. The user may select a protocol and provide input structures by using AstraZeneca identifiers or SMILES strings in the 'input' text field. There is also the option of using a 2D molecular sketcher or to upload SDF files. Furthermore, the number of output conformations can be specified as well as if other information such as calculated properties (e.g., molecular weight, $\operatorname{clog} \mathrm{P}$, various counts, etc.) should be included in the optional output spreadsheet. In this context it should be mentioned that each user has a personalized 'My settings' page. This page provides easy access to customized settings, such as choosing to automatically pre-treat ligand structures by assigning protonation states and enumerating stereoisomers. It also allows the user to specify which (if any) standard 2D calculated properties that will be included in the output.

To further extend the use of 3D-Lab, a web-service was implemented to interface with the data analytics platform D360 [23], using AstraZeneca identifiers. This allows to directly submitting jobs from D360 into 3D-Lab. The ability to easily access, for example, low energy conformations of any compound, may be seen as a significant improvement and helps our vision of shifting medicinal chemists to think of molecules more as $3 \mathrm{D}$ objects than 2D. A general work-flow of 3D-Lab is shown in Figure 3.

The calculated results can be visualized in the output mode; see Figure 4. Visual inspection and further analysis may be performed directly in the output mode, or the generated structure files can be exported 
Research Article Grebner, Norrby, Enström et al.
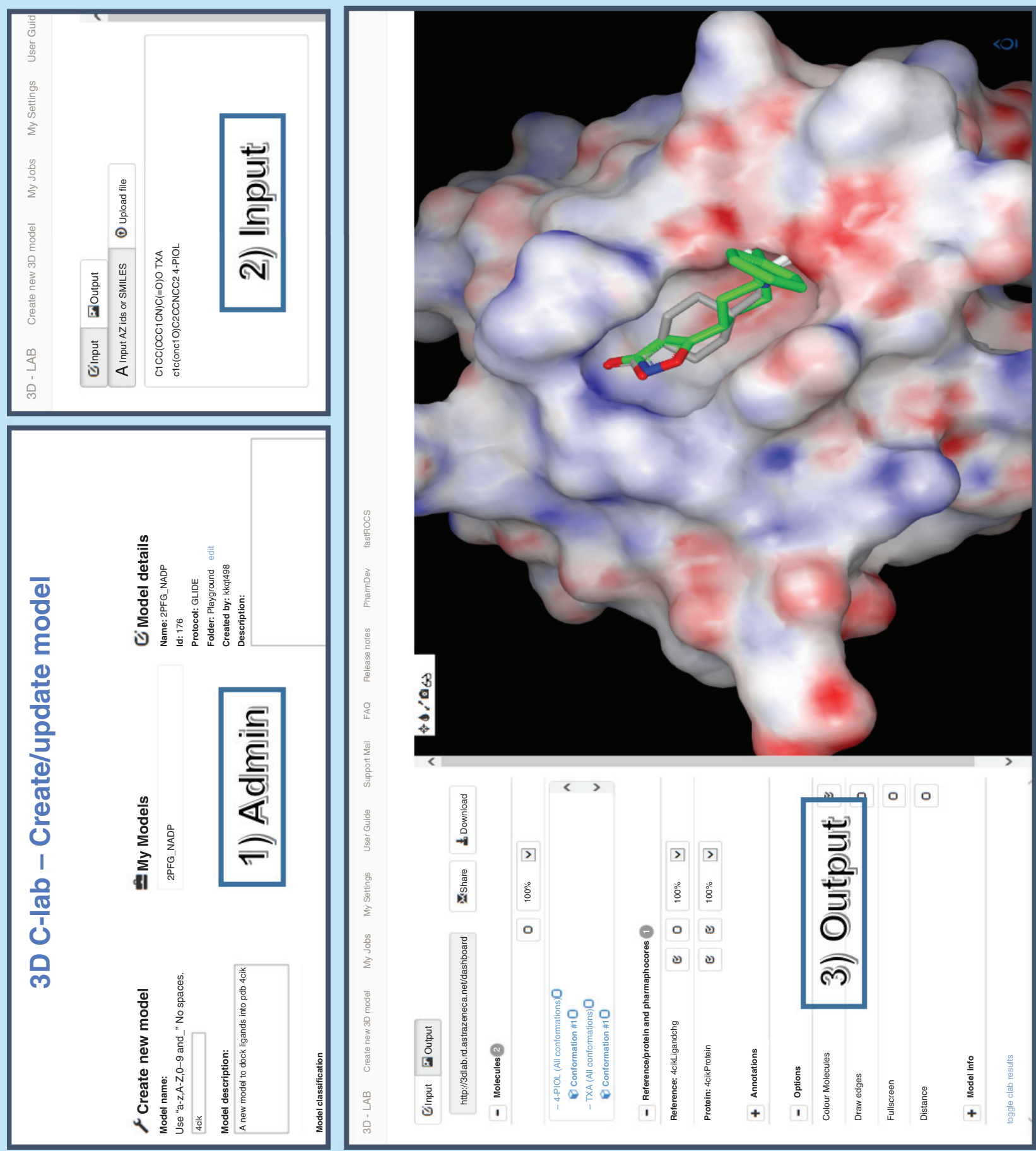

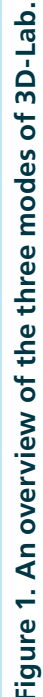




\section{D C-lab - Create/update model}

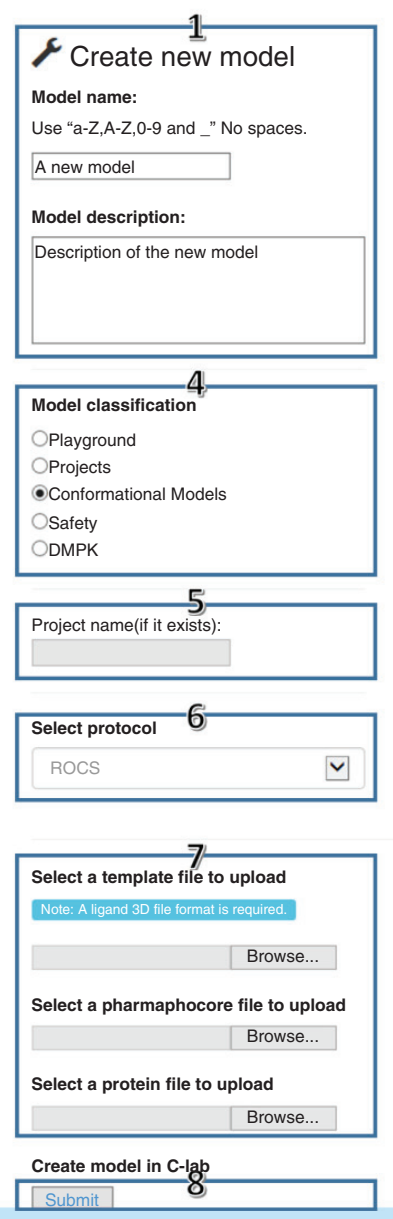

\begin{tabular}{|l|}
\hline My Models \\
2PFG_NADP \\
\hline
\end{tabular}

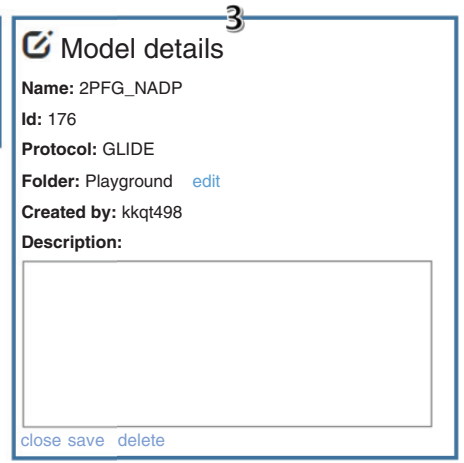

Figure 2. An overview of the admin page. On the left hand side, new models can be created (label 1, 4-8); specifically template files (label 7) are used to define the query molecules, docking grid, the PDB receptor file, and so on, and are uploaded when creating a protocol. The middle panel (label 2) gives an overview of the created models by the current user; the right panel (label 3 ) gives more details about a selected model.

and analyzed elsewhere. Each job is assigned a specific job ID and the results are available via a jobs manager (Figure 4: label 2 - 'My Jobs') and stored in an Oracle database for 6 months, unless otherwise specified. The collaborative aspect is strengthened by allowing sharing of results with colleagues via hyperlinks (using job ids, see Figure 4: labels $7 \& 8$ ). A job can easily be shared by the email functionality. It is important to note that hyperlinks points toward an interactive 3D webpage, and not a static screenshot or the like. In addition, each result's page includes a free-text field providing the possibility to add comments. These annotations are stored in the database and are included in the shared emails (Figure 4).

\section{The JavaScript 3D web visualizer}

The 3D visualizer is developed using HTML5 and JavaScript and employs high-level chemistry components for visualization and data preparation [15]. Previously, 3D viewers for molecular structures in webbrowsers were developed as Java applets (e.g., Jmol [24], AstexViewer [25] and iView [26]). Java apps typically require specific versions of Oracle's Java runtime on each user's computer, which often has been a source of problems. One reason is that users in big organizations (as AstraZeneca) frequently have different versions of Java runtime, possibly because different programs require different Java versions. It does not help that many scientists lack admin rights on their 


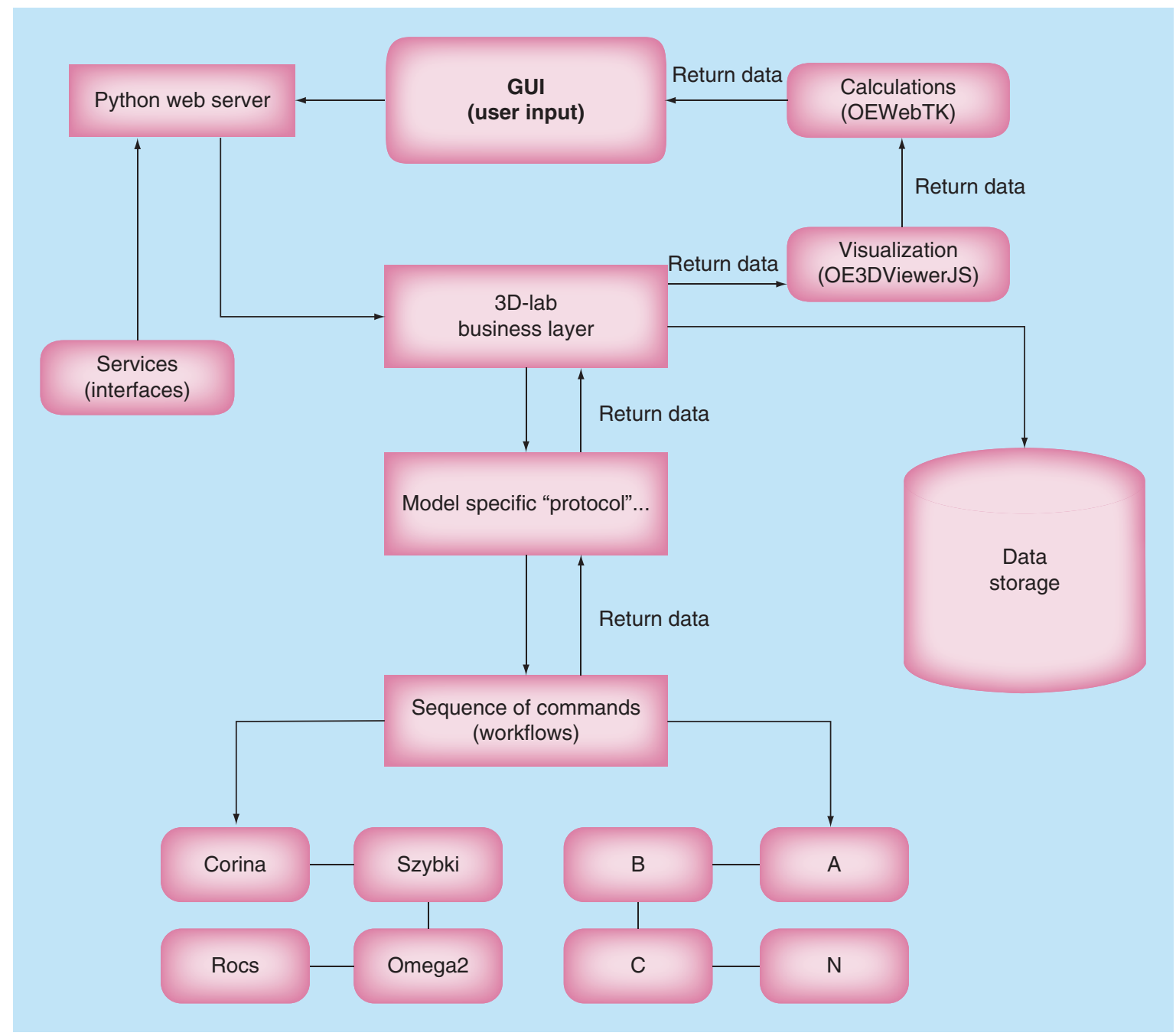

Figure 3. The general work-flow of 3D-Lab. The central GUI communicates with 3D-Lab business layer via a python web-server. From here, all calculations and data management are performed and the results are sent back to the GUI for visualization.

GUI: Graphical user interface.

PCs preventing them from installing/upgrading to the 'correct' version. In addition, Java plug-ins have been a source of many security problems [27]. Thus, we looked for other solutions. Recent browser technology improvements pointed us toward JavaScript. JavaScript is becoming more and more popular, and 'is taking over the world' according to Stack Overflow's annual developer survey [28]. In contrast to Java applets, JavaScript is embedded into the HTML code. It is executed and interpreted during runtime, and it is supported in all current browsers natively. The OpenEye 3D components are based on JavaScript and offer a large number of features (using JSON objects [29]) together with ways of implementing the functions into web services. Figure 4 (label 13) shows an example of the JavaScript $3 \mathrm{D}$ viewer. Most features available in traditional visualizers are available, including the ability for nonspecialists to view $3 \mathrm{D}$ structures in stereo without specific

hardware. As a bonus, publication quality pictures can easily be obtained (by a simple press of a button), a trivial thing previously causing many chemists grief.

There are a few other menus available at the top of the 3D-Lab homepage. In addition to the previously mentioned 'My Jobs' - which keeps track of the user's calculations, 'My Settings' - to personalize settings such as if input molecules should be standardized, there is a 'FAQ' and a 'User Guide' including four brief 'how-to' movies explaining the general work-flow and the three main modes. Finally, there is a link to FastROCS - our virtual screening capability.

\section{Virtual screening capability - FastROCS}

While 3D-Lab is designed to make use of established protocols for design ideas, there is also an interface to a graphics processing unit (GPU) server for virtual screens available from the main homepage. This vir- 


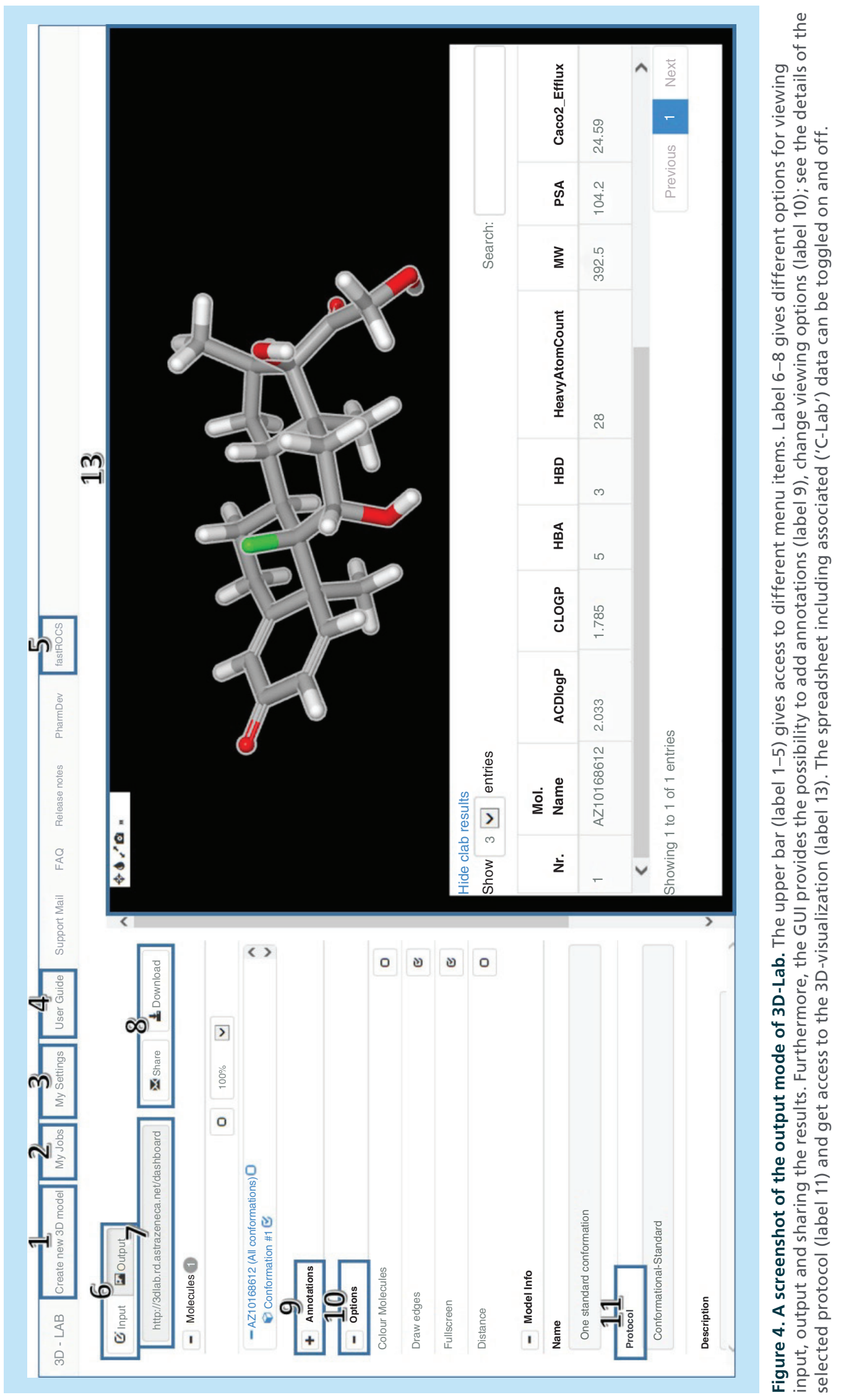


tual screening capability can, for example, be used to identify new lead molecules, but also to serve as an idea generator. All drug designers at AstraZeneca can readily perform virtual screens over multimillionconformer databases to find compounds with similar shape, almost in real-time - just like googling, but searching 3D molecular objects and not text. Somewhat to our surprise, it is frequently used to identify 'nearest neighbors' where traditionally fingerprint methods were used. Thus, apart from being a very efficient and simple way of identifying new shape-similar compounds it facilitates the culture change of looking at chemical structures in 3D instead of 2D. Over the last year, a staggering number of $600+$ virtual screens have been run, indicating its popularity. Having said that, popularity is nice but we of course expect to share impact in the near future. Key to its frequent use and positive feedback is the simple and familiar 'Google-like' interface (Figure 5), in combination with the blazingly fast searches. The dramatic speed-up was achieved by implementing FastROCS, which uses GPU technology for the shape-based molecular alignments [19]. In comparison, FastROCS can search one million conformations per second, as compared with 1000 conformers per second for the original CPU version of ROCS [19], corresponding to a 1000-fold increase in performance.

The FastROCS server is implemented as a web-service, using Flask [30] which is a micro web framework for python. The user starts a virtual screen by provid-

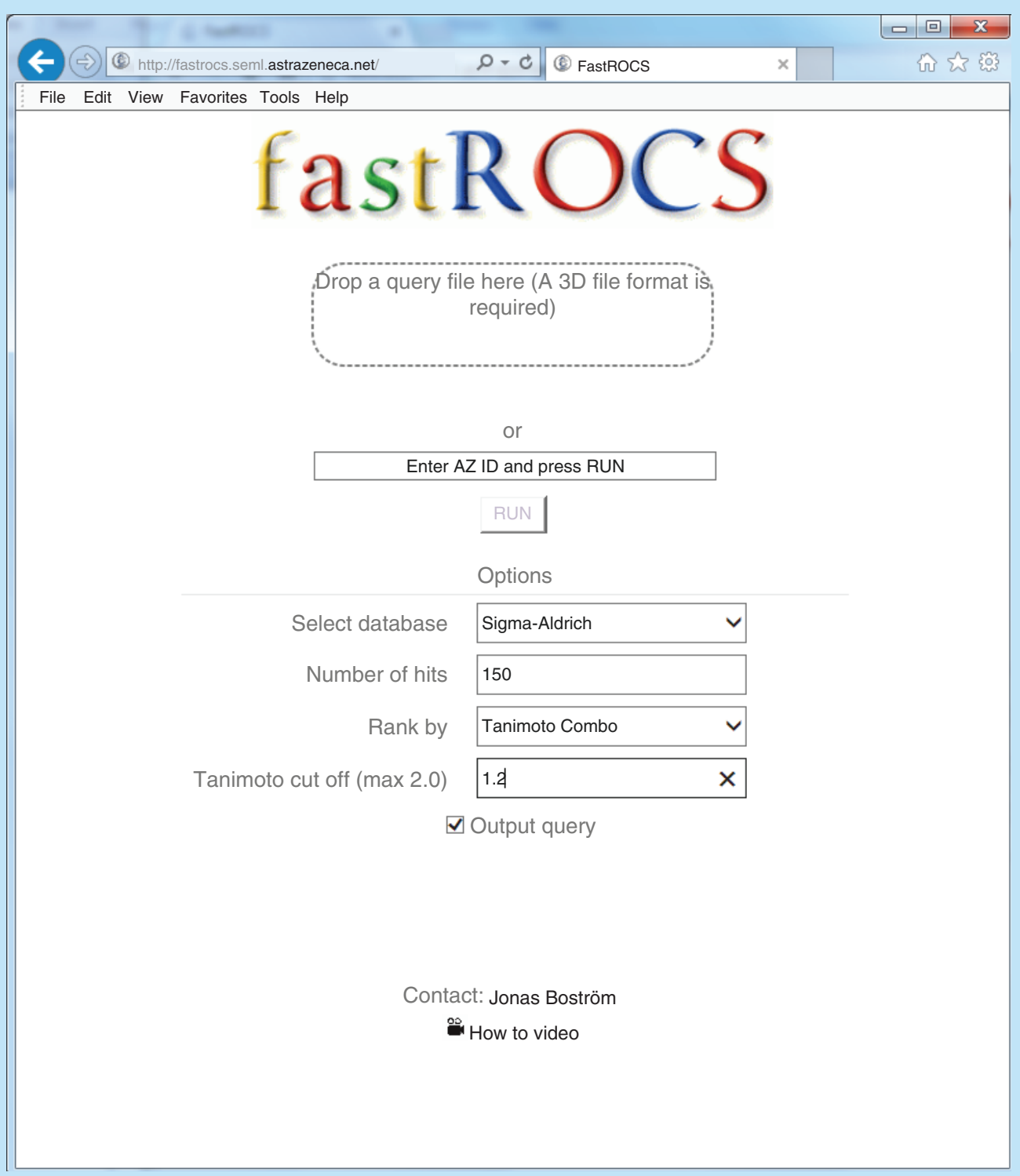

Figure 5. The AstraZeneca 'Google-like' web-based version of FastROCS. 
ing a query molecule. The query molecule can either be loaded by drag-and-drop a molecule file into the search field (typically sdf or mol2, including 3D coordinates) or by supplying an AstraZeneca identifier. If the latter is performed, the web-service goes away and loads the matching input conformation from a precalculated conformational database including all compounds in the corporate screening collection. The user can then choose which database to be searched. Currently four databases are loaded: the AstraZeneca corporate collection; a set of commercially available compounds from Sigma-Aldrich and ligands determined by $\mathrm{x}$-ray crystallography; protein data bank (PDB) [31]; and Cambridge structural database (CSD) [32]. The databases are generated using common OMEGA protocols [33], and are automatically updated on a weekly basis. Moreover, the user has the option to select the number of hits to be reported, which ROCS-specific shape-similarity method (Shape-Only or TanimotoCombo scoring), and which Tanimoto cut-off value should be used (Figure 5). Finally, there is an option to include the query molecule in the results. The results are reported back in three different ways: a tailored pdf document that highlights similarities; an OEB file containing the aligned hits for download; and a link to a web page including the aligned hits. Building on the initial success, upgrades are planned. Among other things, the plans include moving away from the flask server to Django and a RESTful back-end server integration.

\section{Molecular Rift - virtual reality for drug designers}

While the above-mentioned 3D-Lab and FastROCS are designed to make use of well-established protocols in daily drug design, the future work environment and the way of working with 3D data will undoubtedly change. We anticipate that virtual and augmented reality is the future for interacting with $3 \mathrm{D}$ information. A virtual reality for molecular modeling provides a very different perspective, as compared with traditional molecular visualizers. The immersive experience and the feeling of presence change everything. Thus, by exposing the drug designer to a virtual reality a deeper understanding may be achieved, and new ideas could spark which might not have been imagined in a different (and traditional) setting.

Virtual reality was trending in the 1990s, but failed to realize expectations. In the year of 2016, virtual reality is set to take off as this year sees commercial launches of numerous new virtual reality approaches, most using head-mounted devices. For example, Sony launches its PlayStation VR [34], HTC its Vive [35] and the Facebook-owned virtual reality firm Oculus launches the production version of Oculus Rift [36]. In addition, there are simple but elegant solutions like the Samsung Gear VR [37] and the Google Cardboard [38], making it possible to use smartphones for producing a virtual reality experience. The predicted revival of virtual reality can be attributed to its improved performance, increased comfort and accessibility combined with a reasonable price tag.

Recently, we developed the 'next-generation' virtual reality molecular viewer 'Molecular Rift' [14] using the Oculus Rift [36]. It offers a new and exciting way of experiencing small and large molecules, for example ligand-protein complexes, in a virtual reality. By implementing a powerful cheminformatics (open-source) tool-kit [39] and by making the executable and source code available at GitHub free of charge, it received a good amount of attention in social media. With an Altmetric score of 43 , it is currently in the top $5 \%$ of all research outputs scored by Altmetric [40].

That first version of Molecular Rift [14] was controlled with arm and hand motions in combination with finger tracking algorithms using the gaming sensor Microsoft Kinect v2 [41]. The Kinect is designed to track whole bodies, and the resolution for finger tracking was not optimal. Some gestures were considered intricate to learn by users, and the program did not always correctly interpret them. In an attempt to improve the experience with Molecular Rift, we looked into alternatives to enhance the interaction method. The Leap Motion sensor [42], which was recently released, offers higher resolution and accuracy for recognition of fingers and hands and represents a stepchange in gesture control. It was implemented and tested for its usability with respect to increased control. After just a few tests, it was clear that replacing the Kinect with a Leap Motion sensor would improve the user experience of Molecular Rift significantly.

The Leap Motion controller is mounted on the Oculus Rift device (Figure 6A). As a consequence, controlling the viewer becomes independent of the user's orientation and position in the room. This is an advantage compared with the Kinect, where the user had to face the sensor for optimal interaction. Moreover, it was decided to reduce the number of gestures to steer molecules. This was achieved by implementing a small hand controller with four buttons (Figure 6B). Each button activates a specific movement. A few simple and natural interactions can then be performed with the (right) hand, without having to learn any special gestures. The four main actions are: 'translation', 'rotation', 'move camera' and 'reset view'. For example, by pressing the 'rotation' button (using the left hand), the right hand can rotate the molecule. Upon releasing a button, all interactions stop, thus avoiding undesired movements of the molecular objects. User tests 


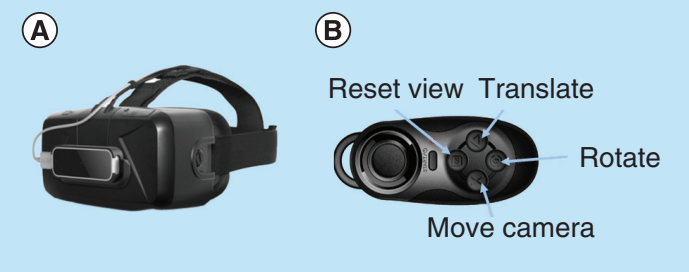

Figure 6. (A) The Leap Motion controller mounted on the Oculus Rift device; (B) the remote virtual reality controller.

showed that the interaction experience was increased by magnitudes and nearly no initial training was required to steer and control the program. As a bonus, by limiting the gestures to the right hand, the left hand was freed up and could be used to activate the in-game menu. That is, when the left hand appears into the user's field of view, an interactive virtual reality in-game menu is activated (see Figure 7A). The in-game menu displays the most common interactions, and pressing the buttons in the virtual reality toggles each option. Furthermore, the in-game menu allows toggling on and off multiple ligands in the input file to visualize, for example, different conformations or ligand poses (Figure 7B). The two-state button 'DemoToggleButton' and the slider control 'DemoSlider' from the Leap Motion core assets Unity widgets [43] were used for this purpose.

In addition to optimizing the interaction method, the virtual reality experience was also improved. By using the Leap Motion infrared camera, an augmented reality was achieved. That is, the surroundings (e.g., room, table) are visualized when the Oculus Rift is directed toward them (Figure 8). This feature allows seeing other people, even when using the goggles, and is one step toward a more collaborative virtual reality. The new improved version of Molecular Rift (version 2) is available free of charge from GitHub: [44]. The interested reader is referred to the original publication for further details on the implementation [14].

\section{Discussion}

By allowing a small group of scientists and programmers with clear goals to act autonomously, development cycles were kept short - 3D-Lab was developed within 1.5 years. The tool is currently gaining traction globally within the AstraZeneca organization. Since the initial launch of 3D-Lab, 60+ users have submitted over 2500 different jobs. The developed platform was designed to be web-based, since browsers are everywhere nowadays and in our experience web-based tools are often perceived as more intuitive than stand-alone programs (doing the same thing).

Why is it advantageous for nonspecialists to apply advanced 3D methods? Well, 3D molecular modeling has been an integral part of drug design since decades. The usage, however, is scattered and often restricted to specialist computational chemists. A proper and straightforward integration of $3 \mathrm{D}$ information and molecular modeling in the daily life of a drug designer is expected to strengthen the in silico capabilities and allow for faster and better decision-making in design cycles. As a consequence, this could reduce the costs of drug discovery, in particular lead discovery, but also lead optimization.

Now and then conservative mindsets are encountered with questions like "Should medicinal chemists really be docking compounds? That's for the experts!" We think otherwise. By exposing well-established 3D information to everyone a general understanding may be achieved as well as pushing for a cultural shift with respect to the extended use of $3 \mathrm{D}$ molecular modeling. That is, molecules are $3 \mathrm{D}$ objects, but are very often treated as $2 \mathrm{D}$ objects. It is important to emphasize that we advocate the use of 3D-Lab in a collaborative sense. The ideal work-flow would be that: a specialist develops a model; the specialist carefully validates it; the model is shared on the web-based platform; the nonspecialist uses it in an explorative fashion; and the specialist and nonspecialist meet and discuss the calculated results and how to best exploit the information in design decisions.

We have stretched the limit far by allowing nonspecialists to run QM methods. QM methods often provide a higher accuracy than empiric methods, but also demand special care since they require a deep understanding of the settings as well as the fact that QM methods are computationally expensive. This may not always apparent for novice users. Therefore, only a few specific and well-established QM protocols, which have previously been proven to work efficiently [20], were implemented. It should be noted that specialists also benefit from having access to simplified protocols for such advanced calculations, freeing up time for other tasks.

Future development of 3D-Lab will include a more flexible admin page, the ability to use a 3D builder and extend the possibility of annotating the 3D results by, for example, bookmarking in sessions. Like all tools (bespoke or commercial) used in discovery, development never stops. There is always room for enhancements, and we anticipate entering a second phase including modifications and integration with other tools. For example, both academia and the pharmaceutical industries invest significant sums in generating $3 \mathrm{D}$ data (e.g., ligand-protein x-ray structures), where the data may be further explored and displayed to broader audience. Thus, a natural step would be to allow users to access PDB-like experimental data through 3D-Lab, while of course carefully monitoring user-experience.

Ligand shape-based approaches, which model physical aspects of molecular recognition, have shown to 
be successful in identifying bioactive compounds in virtual screening in numerous cases. The FastROCS Google-like webpage allowing virtual screens of large databases to identify similar compounds for drug discovery projects using GPU technology has been transformative. We are building on the momentum. In the future, we envision increasing speed, scale and usability. For example, it is a well-known fact that slow Internet searches drive users away. A slow search is considered dissatisfying and gives the impression that the website being broken. This might not sound all that serious but user engagement statistics shows that speed really matters, and the effects are more dramatic than people often imagine. For example, Google have shown that just a 0.5 -s increase in displaying search results caused a $20 \%$ drop in traffic [45]. Thus, in Google's vision to make Internet searches faster speed is now included as a factor in their ranking algorithm. Of course, in drug discovery quality should always triumph speed. But with the recent advances in digitalization, together with significant speed-ups becoming mainstream, the reasons to wait for results over a coffee-break to over-night, as compared with real-time searches, becomes fewer. Google have realized that if a search is fast, users are more likely to search again. This is something we are trying to achieve here as well. That is, we aim to encourage specialists and nonspecialists to try a calculation, get instant feedback and to build on those results.

As an aside, the FastROCS algorithm requires and allows shifting large parts of the computations to
GPUs. These provide a much higher computing density for a lower overall electricity consumption. Most of the HPCs on the Green500 list (the top 500 highperformance energy efficient supercomputers) are built up with GPUs or coprocessors [46]. Thus our FastROCS tool may be termed as green (computational) chemistry [47], as the calculations are much faster thus significantly reducing electricity consumption.

Finally, the development of a virtual reality for drug designers continues to excite us, but we are often encountered with the question of how useful it will be. It remains to be seen if this cool virtual reality environment is more useful than using traditional molecular visualizers. Whether Molecular Rift will be the 'new iPhone' of molecular visualizers or be reduced to some kind of exhibition, something to show-off for 'important' visitors remains to be seen [48]. Nonetheless, traditional visualizers just show molecules in front of you; with Molecular Rift a drug designer can enter a protein-ligand complex. We remove the digital screen and let the user step into, say, a proteinligand complex and to look around. Metaphorically speaking, the improvement may be compared with the one we have seen with regular telephone calls. In the past, we called someone on a telephone using an analog system. Nowadays we often use digital systems (e.g., WiFi technology) and apps like FaceTime or Skype to see and talk to the person we are talking to. In essence, it is the same thing, but a new and significantly improved experience.
(A)

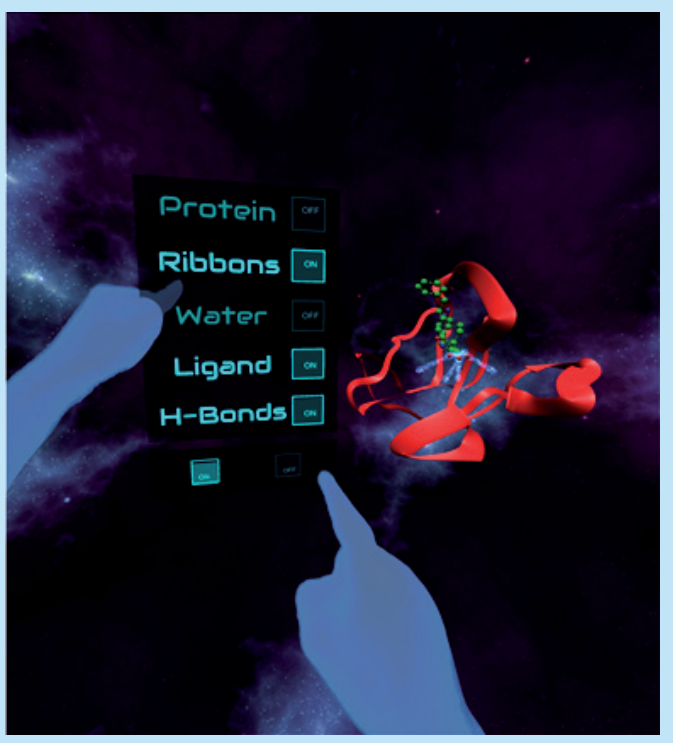

(B)

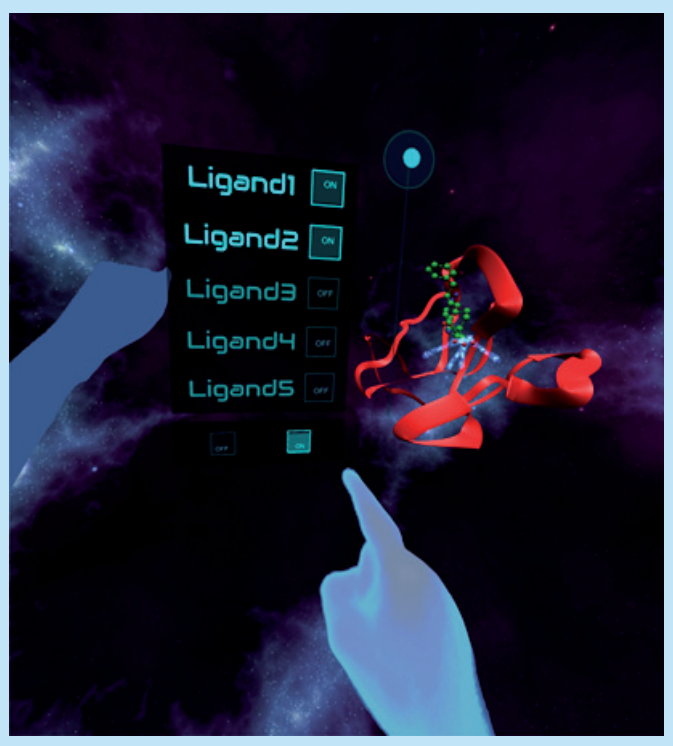

Figure 7. (A) The interactive in-game menu is activated when the left hand appears in the user's field of view. The options are toggled by pressing the virtual reality buttons; (B) multiple ligand structures may be toggled on and off. 


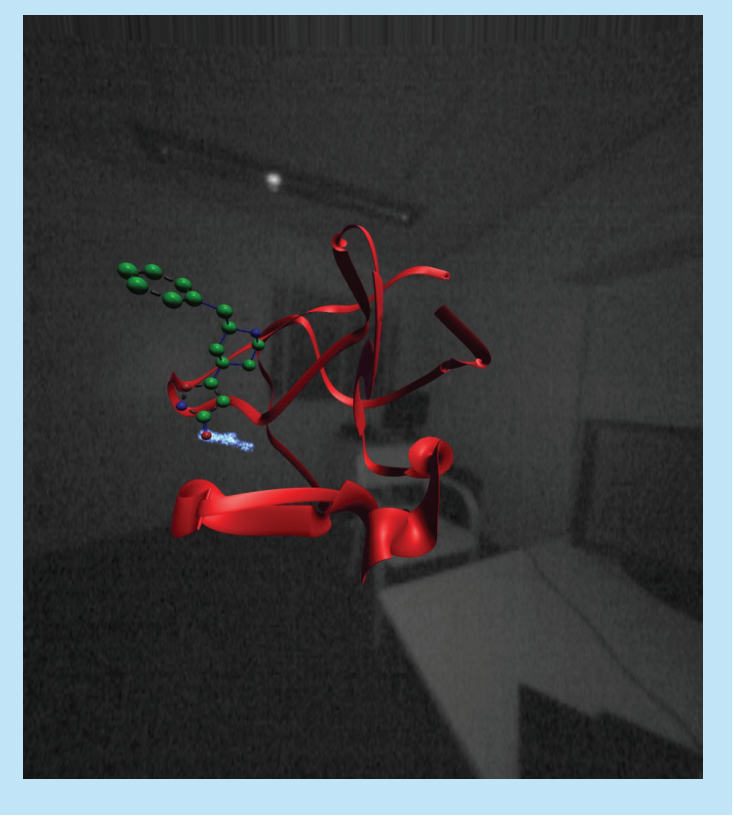

Figure 8. An augmented reality is created using the Leap Motion infrared camera such that the surroundings (e.g., room, table) are visualized when the Oculus Rift is directed toward them.

\section{Future perspective}

Computer visualization and simulations are playing an increasing role in drug design. Future technological developments will speed up data generation (results) and enhance analysis (comprehension). We envision seeing high-quality molecular modeling performed in real-time within 5 years. Needless to say, decision-mak- ing increasingly relies on graphical analysis of data. Users will not accept expert systems that are difficult to use, and will require intuitive easy-to-use tools.

In the near future we will witness full blown augmented virtual reality solutions for all 3D information as well as serving as a virtual meeting room for scientists. Future development for our Molecular Rift includes implementing voice control, and importantly, being able to build and modify the 3D-structures within the virtual reality. With respect to virtual reality and molecular design we predict that Google Cardboard-like apps will show impact [38]. Mobile apps enables virtual reality experiences without having to invest into extensive systems. The traction here is that, unlike headsets like Oculus Rift and HTC Vive, the technology does not need a high-end PC to run applications. Furthermore, apps in a smartphone are mobile in the true sense that no wires, etc., are required. Further benefits of Google Cardboard-like devices are that the hardware may be cheap, if not 'free' (most people already have a smartphone). In this context, we note that the Samsung Gear VR [37] and the coming Google Daydream [49] will be placed somewhere between the simple Google Cardboard and sophisticated Oculus Rift and HTC Vive technology.

Computer systems will capitalize on the consumer market, driving faster developments and a wide adoption allowing the scientist to execute, share and communicate complex simulations and visualizations instantly on the fly with anyone anywhere. In the future, big pharmaceutical companies will have moved their

\section{Executive summary}

- We have developed a collaborative web-app called 3D-Lab. It provides an integrated drug design platform allowing speedier decision-making and more efficient use of 3D information in drug design teams. By integrating the tool into the AstraZeneca high-performance computing and data environment, highthroughput and access to experimental and predicted molecule data are available. 3D-Lab was developed internally by a small and dedicated team within a short time-frame and is now used on a daily basis in live drug design projects. The platform-independent development basically allows using the tool from any Internet-enabled device. The easy way of sharing results facilitates collaborations and aims to motivate discussions of 3D-data within a design team. In essence, 3D-Lab makes 3D information available and usable by whoever and wherever required to impact drug design. The new tool allows easy access to large amount of 3D data (experimental and theoretical) which was previously not trivial to achieve, both for nonspecialists as well as specialist. It is our hope that this possibility will increase the use of 3D information to shape the ideas for future drug-design projects.

- The FastROCS capability, with a Google-like look and feel, allows to perform large-scale ligand-based virtual screen on large multiconformational databases (such as the AstraZeneca compound collection) in a couple of seconds. The new game-changing GPU technology has opened up for science previously not doable, and represents a paradigm shift in 3D shape screening.

- We have improved our virtual reality molecular viewer Molecular Rift for analyzing small and large molecules by implementing the Leap Motion sensor, thereby improving the interaction method. Furthermore, the option to experience an augmented reality has been implemented as well as the in-game menu has been enhanced significantly. Although Molecular Rift may, to date, be seen as a specialized tool in development (but available to everyone from GitHub), we are confident that this way of interacting with molecules will impact the way how future design will be performed. 
infrastructure into the cloud, although among the last enterprises to do so.

\section{Acknowledgements}

The authors thank $T$ Wester (AstraZeneca) for helping provide a creative work environment and J Batista and B Cole (both OpenEye Scientific Software) for providing technical assistance during implementation of FastROCS.

\section{Financial \& competing interests disclosure}

The authors have no relevant affiliations or financial involvement with any organization or entity with a financial

\section{References}

Papers of special note have been highlighted as:

- of interest

1 Riddell FG, Robinson MJT. van't Hoff JH and Le Bel JA - their historical context. Tetrahedron 30, 2001-2007 (1974).

2 Anderson AC. The process of structure-based drug design. Chem. Biol. 10, 787-797 (2003).

3 Ladbury JE. Just add water! The effect of water on the specificity of protein-ligand binding sites and its potential application to drug design. Chem. Biol. 3, 973-980 (1996).

4 Boström J, Grant JA. Exploiting ligand conformations in drug design. In: Molecular Drug Properties - Measurement and Prediction. Mannhold R (Ed.). Wiley-VCH Verlag GmbH \& Co. KGaA Berlin, Hiedelberg, Germany, 183-205 (2008).

5 McGaughey GB, Sheridan RP, Bayly CI et al. Comparison of topological, shape, and docking methods in virtual screening. J. Chem. Inf. Model. 47, 1504-1519 (2007).

6 Hawkins PC, Warren GL, Skillman AG et al. How to do an evaluation: pitfalls and traps. J. Comput. Aided Mol. Des. 22, 179-190 (2008).

7 Brown DG, Boström J. Analysis of past and present synthetic methodologies on medicinal chemistry: where have all the new reactions gone? J. Med. Chem. 59(10), 4443-4458 (2016).

8 Biovia. Virtual biosphere and materials. www.3ds.com/products-services/biovia

9 EnginFrame. www.nice-software.com/products/enginframe

10 Live Design. Collaborative drug design. www.schrodinger.com/livedesign

11 Optibrium. www.optibrium.com

12 Stiefl N, Gedeck P, Chin D et al. FOCUS - development of a global communication and modeling platform for applied and computational medicinal chemists. J. Chem. Inf. Model. 55, 896-908 (2015).

13 FastROCS. www.eyesopen.com/fastrocs

14 Norrby M, Grebner C, Boström J et al. Molecular rift: virtual reality for drug designers. J. Chem. Inf. Model. 55, 2475-2484 (2015).

- An open-source virtual reality molecular viewer using Oculus Rift. interest in or financial conflict with the subject matter or materials discussed in the manuscript. This includes employment, consultancies, honoraria, stock ownership or options, expert testimony, grants or patents received or pending, or royalties.

No writing assistance was utilized in the production of this manuscript.

\section{Open Access}

This work is licensed under the Attribution-NonCommercialNoDerivatives 4.0 Unported License. To view a copy of this license, visit http://creativecommons.org/licenses/by-nc-nd/4.0/

15 OEChem. www.eyesopen.com/oechem-tk

16 Friesner RA, Banks JL, Murphy RB et al. Glide: a new approach for rapid, accurate docking and scoring. 1. Method and assessment of docking accuracy. J. Med. Chem. 47, 1739-1749 (2004)

17 Jones G, Willett P, Glen RC. Molecular recognition of receptor sites using a genetic algorithm with a description of desolvation. J. Mol. Biol. 245, 43-53 (1995).

18 McGann M. FRED pose prediction and virtual screening accuracy. J. Chem. Inf. Model. 51, 578-596 (2011).

19 ROCS.

www.eyesopen.com/rocs

20 Kenny PW. Prediction of hydrogen bond basicity from computed molecular electrostatic properties: implications for comparative molecular field analysis J. Chem. Soc. Perkin Trans. 21, 199-202 (1994)

- A general quantum chemistry approach to predict hydrogen bond strengths.

21 Gaussian 09, Revision E. 01, Frisch MJ, Trucks GW, Schlegel HB et al. Gaussian, Inc., Wallingford, CT, USA (2009).

22 OMEGA. www.eyesopen.com/omega

23 Certara D360. www.certara.com/software/scientific-informatics/d360

24 Jmol. www.jmol.org

25 Hartshorn MJ. AstexViewer: a visualisation aid for structurebased drug design. J. Comput. Aided Mol. Des. 16, 871-81 (2001).

26 Li H, Leung KS, Nakane T, Wong MH. iview: an interactive WebGL visualizer for protein-ligand complex. $B M C$ Bioinf. 15, 56-61 (2014).

27 JavaScript isn't Java. www.howtogeek.com

28 Developer Survey. http://stackoverflow.com/research/developer-survey-2016

29 JSON. http://beta-docs.eyesopen.com/toolkits/cpp/oechemtk/json

30 Flask. http://flask.pocoo.org 
31 Berman HM, Westbrook J, Feng Z et al. The protein data bank. Nucleic Acids Res. 28, 235-242 (2000).

32 Allen F. The Cambridge Structural Database: a quarter of a million crystal structures and rising. Acta Crystallogr. Sect. B 58, 380-388 (2002).

33 Boström J, Greenwood JR, Gottfries J. Assessing the performance of OMEGA with respect to retrieving bioactive conformations. J. Mol. Graphics Model. 21, 449-462 (2003).

34 Sony Playstation VR. www.playstation.com/en-au/explore/playstation-vr

35 HTC Vive.

www.htcvive.com/uk

36 Oculus Rift SDK.

https://developer.oculus.com/

37 Samsung Gear VR.

www.samsung.com/global/galaxy/wearables/gear-vr

38 Google Cardboard. https://vr.google.com/cardboard

39 OpenBabel. http://openbabel.org/wiki
40 Altmetric Score for 'Molecular Rift: Virtual Reality for Drug Designers'.

www.altmetric.com/details/4713779

41 Microsoft Kinect v2.

www.microsoft.com/en-us/kinectforwindows

42 LeapMotion.

www.leapmotion.com

43 Leap Motion Unity Widgets. https://developer.leapmotion.com

44 GitHub: J Bostrom. https://github.com/JBostrom/MolecularRiftv2

45 Google Now Counts Site Speed As A Ranking Factor. http://searchengineland.com

46 The Green500 List - November 2015. www.green500.org/lists/green201511

47 Anastas PT, Warner JC. Green Chemistry: Theory and Practice. Oxford, University Press, NY, USA (1998).

48 This Is Not a Molecular Visualizer. http://blogs.idrugdesign.com/\#post8

49 Google Daydream. https://vr.google.com/daydream/ 